\section{Functional specialization in the supplementary motor complex}

\section{E. Charles Leek and Stephen J. Johnston}

In their recent Review article (Functional role of the supplementary and pre-supplementary motor areas. Nature Rev. Neurosci. 9, 856-869 (2008)) $)^{1}$, Nachev, Kennard and Husain consider data from a broad range of studies involving structures within the supplementary motor complex (SMC) comprising the supplementary motor area (SMA), the supplementary eye fields (SEF) and the pre-supplementary motor area (preSMA). These include studies contrasting self-initiated with externally triggered movements, studies concerning the observation of graspable objects and studies concerning the planning of motor sequences, motor learning and cognitive control (for example, task switching). Nachev et al. suggest that functional characterizations of the SMC may be better cast in terms of a rostrocaudal continuum of graded change in structure and function than in terms of subregions of discrete or modular functional specialization. They discuss one hypothesis, drawn from the data presented, that this continuum of function may be predicted by the complexity of condition-action associations across tasks.

There is one important omission from Nachev et al.'s Review that is of potential theoretical relevance. This comes from the growing body of evidence from functional brain-imaging studies implicating the SMC (along with other brain areas, including the intraparietal sulcus, the right superior parietal lobe and the ventrolateral prefrontal cortex) in abstract mental computations supporting visuospatial transformation in tasks like mental rotation (in which observers judge shape equivalence or mirror reflection across changes in visual-stimulus orientation $)^{2-6}$. Of interest also is that visuo-spatial processing in tasks like mental rotation has been shown to be impaired in (at least some) patients with Parkinson's disease $\mathrm{e}^{7-9}$, the underlying pathology of which, as discussed by Nachev et al., is known to affect the SMC - for example, via the loss of pyramidal neurons in the pre-SMA and via dopamine depletion in the nigro-striatal pathways linking the basal ganglia and the pre-motor cortex $^{10}$. The functional association between visuo-spatial transformation and motor processing has been further demonstrated in behavioural studies showing interference costs during the simultaneous performance of manual- and mental-rotation tasks ${ }^{11-13}$.

These findings support a functional association between the SMC and visuospatial transformation that, in agreement with Nachev et al., suggests that the SMC has a function beyond the planning and control of visually guided behaviour. However, it is not clear how SMC involvement in visuospatial transformation can be accounted for in terms of the differential complexity of condition-action associations, where this is conceptualized in relation to stimulusresponse mappings. Of potential relevance is that visuo-spatial transformation does potentially share other computational properties with tasks related to the planning and control of movement that have previously been shown to involve the SMC. One possible link relates to what has been described as "motor simulation"6 of mental rotation or visuo-spatial transformation. A more specific computational hypothesis is that visuospatial transformation and activities related to movement planning and control (for example, sequence learning and reaching) are associated by an underlying functional reliance on vector transformation ${ }^{2,14,15}$. That is, any form of directional motor activity can be assumed to involve the calculation of a vector that specifies the direction and distance required to effect limb movement from one location in space to another (for example, towards a new target). Similarly, the determination of stimulus orientation in space, and of the angular transformation required to remap feature locations from one coordinate system to another, can also be assumed to involve calculation of a vector. According to this hypothesis, regions of the SMC (possibly the pre-SMA ${ }^{2,4}$ ) may be functionally involved in the computation of abstract vector transformations such as those that have been associated with neuronal-population vector transformations underlying the planning of movement ${ }^{14}$ and with visuo-spatial image normalization during mental rotation ${ }^{2,15}$. This characterization proposes a relatively abstract computational role within the SMC beyond the traditional view. In addition, it defines and predicts a domain of functional involvement of the SMC in a diverse range of cognitive tasks requiring the computation of vector-based transformations that will include the preparation for limb movement in response to internal or external cues, the learning of motor sequences and the suppression of these activities via 'cognitive' control.

E. Charles Leek and Stephen J. Johnston are at the Centre for Clinical and Cognitive Neuroscience, School of Psychology, Bangor University, Bangor, Gwynedd, LL57 2AS, Wales.

Correspondence to E.C.L. e-mail:e.c.leek@bangor.ac.uk

1. Nachev, P., Kennard, C. \& Husain, M. Functional role of the supplementary motor and pre-supplementary motor areas. Nature Rev. Neurosci. 9, 856-869 (2008).

2. Johnston, S. J, Leek, E. C., Atherton, C. J., Thacker, N. A. \& Jackson, A. Functional contribution of medial pre-motor cortex to visuo-spatial transformation in humans. Neurosci. Lett. 355 209-212 (2004).

3. Richter, W. et al. Motor area activity during mental rotation studied by time resolved single trial fMRI. J. Cogn. Neurosci. 12, 310-320 (2000)

4. Windischberger, C., Lamm, C., Bauer, H. \& Moser, E. Human motor cortex activity during mental rotation. Neuroimage 20, 225-232 (2003).

5. Vingerhoets, G. et al. Regional brain activity during different paradigms of mental rotation in healthy volunteers: a position emission tomography study. Neuroimage 13, 381-391 (2001).

6. Zacks, J. M. Neuroimaging studies of mental rotation: a meta-analysis and review. J. Cogn. Neurosci. 20, 1-19 (2008).

7. Amick, M. H., Schendan, H. E., Ganis, G. \& Cronin-Golomb, A. Frontostriatal circuits are necessary for visuomotor transformation: mental rotation and Parkinson's disease. Neuropsychologia 44, 339-349 (2006).

8. Crucian, G. P. et al. Mental object rotation in Parkinson's disease. J. Int. Neuropsychol. Soc. 9 1078-1087 (2003).

9. Lee, A. C., Harris, J. P. \& Calvert, J. E. Impairments of mental rotation in Parkinson's disease. Neuropsychologia 36, 109-114 (1998)

10. MacDonald, V. $\&$ Halliday, G. M. Selective loss of pyramidal neurons in the presupplementary motor cortex in Parkinson's disease. Mov. Dis. 17, 1166-1173 (2002).

11. Wohlschlager, A. \& Wohlschlager, A. Mental and manual rotation. J. Exp. Psychol. Hum. Percept. Perform. 24, 397-412 (1998).

12. Wohlschlager, A. Mental object rotation and the planning of hand movements. Percept. Psychophys. 63, 709-718 (2001).

13. Wexler, M., Kosslyn, S. M. \& Berthoz, A. Motor processes in mental rotation. Cognition 68, 77-94 (1998).

14. Georgopoulos, A. P. \& Pellizzer, G. The mental and the neural: psychological and neural studies of mental rotation and memory scanning. Neuropsychologia 33 1531-1547 (1995).

15. Pellizzer, G. \& Georgopoulos, A. P. Common processing constraints for visuomotor and visual mental rotations. Exp. Brain Res. 93, 165-172 (1993). 